\title{
Inflammatory parameters must always be taken into account in ST-segment elevation myocardial infarction
}

We have read the article "Does SYNTAX score II predict poor myocardial perfusion in ST-segment elevation myocardial infarction?" by Wang et al. [1] with great interest. The authors concluded that high SYNTAX score II is an independent predictor of myocardial blush grade (MBG) 0/1 in patients with ST-segment elevation myocardial infarction (STEMI) undergoing primary percutaneous coronary intervention (pPCI).

There is no doubt that MBG is predictive of outcome in patients with an acute STEMI undergoing pPCI [2]. MBG was also an independent predictor of mortality even after adjusting for the presence of Thrombolysis in Myocardial Infarction (TIMI) flow grade 3, the corrected TIMI frame count, and other clinical parameters [2]. Recently it has been shown that the neutrophil to lymphocyte ratio was associated with impaired myocardial perfusion and adverse long-term outcome in patients with STEMI undergoing pPCI [3].

SYNTAX score II consisted of two anatomical (SYNTAX score and unprotected left main coronary artery disease) and six clinical variables (age, creatinine clearance, left ventricular ejection fraction, sex, chronic obstructive pulmonary disease, and peripheral vascular disease) [4]. Therefore, we are convinced that if inflammatory parameters were regarded as a covariate in addition to SYNTAX score II variables, the study could completely reflect the effect of SYNTAX score II on myocardial perfusion in STEMI.

Conflict of interest: None declared

\section{References}

1. Wang G, Hua Q, Li J et al. Does SYNTAX score II predict poor myocardial perfusion in ST-segment elevation myocardial infarction? Cardiol J, 2016; 23: 317-323. doi: 10.5603/CJ.a2016.0006.

2. Gibson CM1, Cannon CP, Murphy SA, Marble SJ, Barron HV, Braunwald E. Relationship of the TIMI myocardial perfusion grades, flow grades, frame count, and percutaneous coronary intervention to long-term outcomes after thrombolytic administration in acute myocardial infarction. Circulation, 2002; 105: 1909-1913.

3. Sen N, Afsar B, Ozcan F et al. The neutrophil to lymphocyte ratio was associated with impaired myocardial perfusion and long term adverse outcome in patients with ST-elevated myocardial infarction undergoing primary coronary intervention. Atherosclerosis, 2013; 228: 203-210.

4. Dibra A, Mehilli J, Dirschinger J et al. Thrombolysis in myocardial infarction myocardial perfusion grade in angiography correlates with myocardial salvage in patients with acute myocardial infarction treated with stenting or thrombolysis. J Am Coll Cardiol, 2003; 41: 925-929.

Sinan İscen

Diyarbakır Military Hospital, Diyarbakır, Turkey e-mail:dr.iscen@hotmail.com 